\title{
Halting Schistosoma haematobium - Associated Bladder Cancer
}

\author{
Monica C. Botelho, ${ }^{1,2,}{ }^{*}$ Helena Alves, ${ }^{2}$ and Joachim Richter ${ }^{3}$ \\ ${ }^{1}$ INSA, National Institute of Health Dr. Ricardo Jorge, Porto, Portugal \\ ${ }^{2}$ I3S, Instituto de Investigacao e Inovacao da Universidade do Porto, Portugal \\ ${ }^{3}$ Institute of Tropical Medicine and International Health, Charite - Universitatsmedizin Berlin, Germany \\ "Corresponding author: Monica C. Botelho, INSA - National Institute of Health Dr. Ricardo Jorge, Rua Alexandre Herculano, 321, 4000-055, Porto, Portugal. Tel: +351-223401114; \\ Fax: +351-223401109, E-mail: monicabotelho@hotmail.com
}

Received 2016 October 21; Revised 2016 December 07; Accepted 2017 September 16.

\begin{abstract}
Context: At present schistosomiasis is endemic in 78 countries, affecting more than 260 million people. Schistosomiasis haematobia alone affects more than 112 millions.

Evidence Acquisition: We performed a computerized search of in PubMed database with keywords: Bladder Cancer Cost and Schistosomiasis Mass Treatment.

Results: Bladder cancer is an important sequelae of this infection. In low-resource countries, where this disease is endemic, individuals inflicted with bladder cancer have very limited access to treatment and death is most probably certain.

Conclusions: Mass treatment with praziquantel is an easy, safe, and inexpensive treatment that could save the lives of thousands and reduce the morbidity of millions.
\end{abstract}

Keywords: Schistosomiasis, Bladder, Cancer, Mass Treatment

\section{Context}

Schistosomiasis, is an ancient scourge of mankind, depicted in papyri from Pharaonic Egypt and known from human remains over 2000 years from China. Human schistosomiasis is caused by six species: Schistosoma (S.) haematobium, S. mansoni, S. japonicum, S. intercalatum, S. guineensis, S. mekongi. A further species S. malayensis has been identified to infect humans in a limited focus in Malaysia (1).

Schistosomiasis was originally called bilharziasis in homage to Theodor Bilharz, a young German pathologist, who first described the disease and its association with Schistosoma worms, working at Cairo in Egypt. Blood-dwelling Trematoda (phylum Platyhelminthes) of the genus Schistosoma cause this chronic and debilitating disease (2). Schistosomes present a heteroxenic life cycle, requiring as secondary host an invertebrate freshwater snail. Geographic distribution and maintenance of human infection depend on the presence of a suitable snail host. Adult worms are sexually distinct, a characteristic that separates them from other flukes that are hermaphrodites. Males present a ventral infolding from the ventral sucker to the posterior end forming the gynecophoric canal, where females are kept during copulation (copulatory groove). Schistosoma life cycle differs from that of other flatworms also by infecting process of the definitive host: the penetration of cercariae through the skin. Of the Schistosoma species pathogenic to humans, only Schistosoma haematobium causes urinary schistosomiasis (3). Snails of the genus Bulinus are the principle intermediate hosts for S. haematobium. Animals may play a role in S. haematobium infections as they may hybridize with closely-related zoonotic Schistosoma spp. such as $S$. bovis (4).

As of 1989 schistosomiasis was endemic in 76 countries (5). Recent World Health Organization (2) report declared that schistosomiasis is endemic in 78 countries $(6,7)$. The estimated total number of people requiring treatment for schistosomiasis in 2013 was 261008 019, of whom 121170 936 (46.4\%) were school-aged children ( $5-14$ years) (7).

S. haematobium is endemic in 53 countries in the Middle East and most of the African continent, including the islands of Madagascar and Mauritius. Due to successful eradication programs, the infection is no more of significant public health significance in Egypt, Lebanon, Oman, Syria, Tunisia, and Turkey, because transmission is low or nonexistent. A disputed and ill-defined focus exists in India and requires further confirmation (2). After more than 50 years, in which no more autochthonous cases of schistosomiasis were recorded in Europe, S. haematobium infection has recently emerged in Corsica (8).

In this review, we discuss the basis for making benefi- 
cial therapeutic purposes in prevention, treatment, and reducing the effects of schistosomiasis, such as bladder cancer mortality.

\section{Evidence Acquisition}

In this article, the cost of bladder cancer treatment and the success of mass treatment for eradicating schistosomiasis are reviewed. For the literature review, we have used standard search strategies involving querying PubMed online database, using key terms including "bladder cancer cost" and "schistosomiasis mass treatment". No specific key words were required as inclusion criteria. The reference lists of each article have been reviewed, in details, to find additional articles. Articles found were reviewed independently in full text.

\section{Results}

\subsection{Schistosomiasis-Associated Bladder Cancer}

Squamous cell carcinoma of the bladder (SCC) is a malignant, poorly differentiated neoplasm. SCC is the common form of bladder cancer in rural Africa, where S. haematobium is prevalent $(9,10)$. By contrast, the majority of bladder cancer in developing countries and regions not endemic for urogenital schistosomiasis is transitional cell carcinoma (TCC), which arises from the transitional epithelium lining of the bladder. The parasite eggs trapped in the bladder wall release antigens and other metabolites (presumably evolved to expedite eggs to the urine, and hence to the external environment). The phenomenon leads to hematuria and to chronic inflammation, increasing the risk of SCC of the bladder. The epidemiological association between SCC of the bladder with schistosomiasis haematobia is based both on case control studies and the correlation of bladder cancer incidence with the prevalence of $S$. haematobium infection within diverse geographic areas. The incidence of urogenital schistosomiasis-associated SCC is estimated in 3 to 4 cases per 100 000, yearly (11). Schistosomiasis haematobia is a chronic infection. The adult, egg-producing schistosomes live for many years, re-infections frequently occur, and schistosomiasis-associated bladder SCC appears relatively early, often by the mid-decades of life (TCC usually presents in the later decades of life). In its recent monograph, IARC (International Agency for Research on Cancer) confirmed that chronic infection with S. haematobium causes the cancer of urinary bladder (12).

Our group has been working on the identification of parasite derived compounds that might be implicated in the carcinogenesis of S. haematobium. The majority of these compounds are catechol estrogens. The genotoxic effects of these estrogen metabolites might be attributed to oxidation of catechol estrogens to quinones, followed by redox cycling and formation of reactive oxygen species that, in turn, react with DNA (13). Given the context of the unarguable link between $S$. haematobium infection and bladder cancer, the presence of putative carcinogenic molecules in S. haematobium eggs hopefully may have practical consequences for new approaches to disease control $(14,15)$. The metabolism of estrogens and production of depurinating estrogen-DNA adducts can be implicated in a pathway underlying $S$. haematobium-promoted host cell DNA damage, leading eventually to cell transformation. The carcinogenic effect of this estrogen-DNA adduct mediated pathway could explain the link between chronic schistosomiasis haematobia and SCC of the bladder. We anticipate that these findings will contribute to understand how schistosomiasis haematobia leads to SCC of the bladder (13).

\subsection{Treatment Cost of Bladder Cancer}

Although the costs of medical care for bladder cancer have been investigated extensively, patient time costs associated with cancer care have rarely been estimated systematically. Yabroff and colleagues (16) identified 763,527 US patients with cancer from 1995 to 2001 . Net time cost estimates for the initial phase of care were applied to national estimates of numbers of new cancers in 2005 to obtain national time costs for the initial phase of care. According to these authors in 2005, patient time costs for the initial phase of care were 2.3 billion dollars (16).

In another example, the diagnosis and treatment of bladder cancer represents a significant financial burden to the population in the United States. Therapeutic advances in bladder cancer care have come at a high cost to payers, providers, and patients. In this study, Noyes et al. (17) describe the principles of cost-effectiveness evaluation in healthcare and provide recommendations for a more economical use of resources in bladder cancer care. Although several studies have demonstrated that bladder cancer is a common disease associated with substantial economic burden for patients and society, the evidence supporting the cost-effectiveness of many interventions in bladder cancer care is limited and of insufficient quality. In addition, very little is known about the quality of life and the preferred outcome measure for economic evaluations associated with bladder cancer states and treatments. Moreover, current clinical guidelines for bladder cancer care do not incorporate economic factors while evaluating clinical pathways (17).

In Canada, bladder cancer is the most costly malignancy to treat per patient. Treatment options for bladder 
cancer are immediate cystectomy and conservative therapy with intravesical Bacillus Calmette-Guerin (BCG). The corresponding mean per-patient discounted lifetime costs (in 2005 Canadian dollars) were $\$ 37,600$ and $\$ 42,400$, respectively (18). In 2006, the annual cost of care for all patients with muscle-invasive BC (MIBC) was $\$ 35.72 \mathrm{M}$, 70\% more than the $\$ 21.03 \mathrm{M}$ for patients with non-MIBC. The major cost drivers, regardless of disease stage, were diagnostic/surveillance and complications, accounting for up to $43 \%$ and $37 \%$ of BC care costs, respectively. Co-morbidityadjusted incremental annual resource costs per patient with MIBC were more than 4 times greater than those for patients with non-MIBC, similar to those of OC controls ( $P$ $=0.490-0.913)$, except for inpatient $(\mathrm{P}=0.002)$ and hospice $(\mathrm{P}<0.001)$ costs, which were both statistically significantly lower. Annual adjusted incremental Medicare reimbursements totalled \$36.3M for non-MIBC and \$96.1 million for MIBC (19).

In 2007, Konety and Allareddy (20) identified 6577 American patients undergoing radical cystectomy for bladder cancer from the Nationwide Inpatient Sample of the Healthcare Cost and Utilization Project (1998 to 2002). Of commonly performed urological cancer procedures, radical cystectomy is associated with the highest morbidity and mortality. According to these authors, the impact of each individual type of complication or a combination of them on various outcome measures, such as mortality, charges, and length of stay is unclear. They attempted to quantify the impact of specific post-cystectomy complications and combinations thereof in terms of mortality, charges, and length of stay. Although most patients undergoing cystectomy are older and have multiple comorbidities, the postoperative complications with the most significant impact were those directly related to surgery (primary complications). Secondary, complications (cardiac, respiratory, vascular, etc) appear to have less of an impact on most common outcome measures. The median total charge was 41,905 dollars and median length of stay was 9 days (20).

The calculations of treatment costs are limited by the fact that SCC may cause different costs than TCC and that costs depend on the current exchange rate of a country's currency with the USD. However, figures allow an approximate estimation of costs SCC would produce in industrialized countries if properly treated according to current standards.

\subsection{Mass Treatment of Schistosomiasis and Preventive Chemotherapy}

It has been previously shown that in patients treated with praziquantel, pathological lesions of the urinary tract disappear, as shown ultrasonographically (21). In these patients, the resolution of bladder lesions induced by urinary schistosomiasis resulted in reversibility of urinary tract obstructions 1 year after treatment with praziquantel (2224). Other chronic squeal like Symmers' fibrosis has been shown to be reversed by praziquantel (25).

We anticipate that the incidence of SCC in endemic areas will decrease to that of non-endemic regions if patients with urinary schistosomiasis are regularly treated from childhood on, in spite of constant re-exposure, as it has been demonstrated $(6,7)$.

The literature provides us with several reports, in which mass treatment with praziquantel with the purpose of preventive chemotherapy of schistosomiasis is effective. An intervention study was conducted in Khamir, north of Sana'a Yemen, for control of urinary schistosomiasis, using chemotherapy. The prevalence of S. haematobium infection 14 months post-intervention fell from $58.9 \%$ to $5.8 \%$ and frequency of heavy infection from $40.0 \%$ to $18.9 \%$ (26). Schistosomiasis and soil-transmitted helminthiasis (STH) are among the neglected tropical diseases in Africa. A national control program for these diseases was initiated in Uganda during March 2003. Annual treatment with praziquantel and albendazole was given to school children in endemic areas and adults in selected communities, where local prevalence of S. mansoni in school children was high. Two rounds of treatment significantly reduced the prevalence of S. mansoni infection in school children across 3 regions in the country from $33.4 \%-49.3 \%$ to $9.7 \%-29.6 \%$, and intensity of infection from 105.7 - 386.8 eggs per gram of faeces (epg) to 11.6 - 84.1 epg. The proportion of children with heavy $S$. mansoni infection was significantly reduced from $15 \%$ (95\% CI 13.4\%-16.8\%) to $2.3 \%$ (95\% CI 1.6\%$3.0 \%$ ). In the current study, the authors showed that annual antihelminthic treatment delivered to school children and adults at high risk in Uganda could significantly reduce the prevalence and intensity of infection for schistosomiasis and STH, and both potentially and significantly reduce the levels of environmental transmission of infection (27).

Likewise, in case of genital infection with S. haematobium, it was shown that preventive chemotherapy could be useful. Genital infection with S. haematobium is prevalent in sub-Saharan Africa. Epidemiological studies have observed that genital schistosomiasis is associated with an increased odd of HIV infection among women. Ndeffo Mbah et al. (28) estimated that, in S. haematobium high-risk communities, targeted annual treatment of school-aged children could reduce HIV prevalence by $20 \%$ (95\% CI: $12 \%-31 \%$ ) in Angola, 16\% (95\% CI: 10\%-32\%) in Kenya, and 6\% (95\% CI: $3 \%-18 \%)$ in Zambia after the first 20 years of intervention (28).

The latest success story in the literature was designed 
to assess the impact of a decade of biennial mass administration of praziquantel on schistosomiasis in school-aged children in Burkina Faso. In 2013, in a national assessment based on 22 sentinel sites, 3514 school children aged between 7 to 11 years were checked for S. haematobium and S. mansoni infection by the examination of urine and stool samples, respectively. Ouedraogo et al. (29) analysed the observed prevalence and intensity of infections and compared these with the relevant results of earlier surveys in Burkina Faso. Less than 1\% of the children in 6 regions had heavy S. haematobium infections. S. mansoni was only detected in 2 regions. By mass use of preventive chemotherapy, Burkina Faso may have eliminated schistosomiasis as a public health problem in 8 regions and controlled schistosome-related morbidity in other 3 regions (29).

On the other hand, the number of people treated for schistosomiasis worldwide in 2013 was 39485376 . This represents only $12.7 \%$ of the population requiring preventive chemotherapy for schistosomiasis globally. Schistosomiasis treatments in 2013 were reported by 9 fewer countries than in 2012. In 2013, 160 million praziquantel tablets were delivered to countries in the African Region for the implementation of treatment. This would have been enough to treat about 60 million people. The fact that little more than 30 million people were treated in 2013 suggests that the availability of praziquantel was not the only limiting factor for schistosomiasis control in the region (7). Considering that there are at least 112 million people infected with schistosomiasis haematobia $(13,30-32)$, and given that 3 to 4 in 100000 individuals yearly develop bladder cancer (see above), the incidence of SCC attributable to schistosomiasis expected would approximately amount to 5000 patients yearly with bladder cancer caused by S. haematobium infection worldwide. The bladder cancer treatment of these patients would cost at least 20 million USD per year (see above).

In contrast, given that the individual cost per treatment including logistic costs amounts to 0.32 USD and that a biennial treatment is necessary to prevent serious morbidity due to schistosomiasis, the annual cost per schistosomiasis patient amounts to 0.16 USD (33). Mass therapy which prevents SCC in schistosomiasis patients would than cost 17.92 million USD per year and a chronic often incurable disease like SCC would be not only "treated" but safely prevented (34). If only school-aged children (46.4\%; 5 - 14 years) were targeted, the cost would drop to 9.61 million USD. Affected populations would benefit from other effects of praziquantel on other complications and sequeleae of schistosomiasis, including renal impairment, increased transmission of HIV, HBV, HTLV and other STDs sterility, complicated pregnancy, bleeding from esophageal varices, to mention just a few (35-37).

\section{Conclusions}

Schistosomiasis is a neglected tropical disease, which in early stages is a disease easy to treat and, thus, to eliminate schistosomiasis induced SCC. The problem to solve is political rather than medical. More commitment is urgently needed to tackle this disease. Leading policy makers should be acquainted with these data. Are there simpler and more cost-effective ways of combating cancer than that to eliminate schistosomiasis induced SCC by preventive praziquantel mass therapy? Preventing schistosomiasis is the best treatment and the least expensive way of fighting bladder cancer in endemic areas.

\section{Acknowledgments}

The authors would like to thank the millions of people infected with schistosomiasis, who inspired us to study this disease.

\section{Footnotes}

Authors' Contribution: None declared.

Conflict of Interests: None declared.

Financial Disclosure: None declared.

\section{References}

1. Adamson PB. Schistosomiasis in antiquity. Med Hist. 1976;20(2):17688. [PubMed: 781425]

2. Steinmann P, Keiser J, Bos R, Tanner M, Utzinger J. Schistosomiasis and water resources development: systematic review, meta-analysis, and estimates of people at risk. Lancet Infect Dis. 2006;6(7):411-25. doi: 10.1016/S1473-3099(06)70521-7. [PubMed:16790382].

3. Botelho MC, Machado JC, Brindley PJ, Correia da Costa JM. Targeting molecular signaling pathways of Schistosoma haemotobium infection in bladder cancer. Virulence. 2011;2(4):267-79. [PubMed: 21788729].

4. Mone H, Holtfreter MC, Allienne JF, Mintsa-Nguema R, Ibikounle $\mathrm{M}$, Boissier J, et al. Introgressive hybridizations of Schistosoma haematobium by Schistosoma bovis at the origin of the first case report of schistosomiasis in Corsica (France, Europe). Parasitol Res. 2015;114(11):4127-33. doi: 10.1007/s00436-015-4643-4. [PubMed: 26268566]

5. Mott KE. Contrasts in the control of schistosomiasis. Mem Inst Oswaldo Cruz. 1989;84 Suppl 1:3-19. [PubMed: 2517866].

6. World Health Organization . Weekly epidemiological record, 90th year; 30 January 2015. 90. ; 2015.

7. WHO . Schistosomiasis: progress Report 2001-2011, strategic plan 2012-2020. Geneva: World Health Organization; 2013.

8. Holtfreter MC, Moné H, Müller-Stöver I, Mouahid G, Richter J. Schistosoma haematobium infections acquired in Corsica, France, August 2013. Euro Surveill. 2014;19(22):20821.

9. Mostafa MH, Sheweita SA, O'Connor PJ. Relationship between schistosomiasis and bladder cancer. Clin Microbiol Rev. 1999;12(1):97-111. [PubMed: 9880476]. 
10. Zhong X, Isharwal S, Naples JM, Shiff C, Veltri RW, Shao C, et al. Hypermethylation of genes detected in urine from Ghanaian adults with bladder pathology associated with Schistosoma haematobium infection. PLoS One. 2013;8(3):e59089. doi: 10.1371/journal.pone.0059089. [PubMed: 23527093].

11. Shiff C, Veltri R, Naples J, Quartey J, Otchere J, Anyan W, et al. Ultrasound verification of bladder damage is associated with known biomarkers of bladder cancer in adults chronically infected with Schistosoma haematobium in Ghana. Trans $R$ Soc Trop Med Hyg 2006;100(9):847-54. doi: 10.1016/j.trstmh.2005.10.010. [PubMed: 16443246].

12. IARC Biological agents, A review of human carcinogens. IARC monographs on the evaluation of carcinogenic risks to humans/WHO. IARC. 2012;100(Pt B):1-441.

13. Botelho MC, Alves H, Barros A, Rinaldi G, Brindley PJ, Sousa M. The role of estrogens and estrogen receptor signaling pathways in cancer and infertility: the case of schistosomes. Trend Parasitol. 2015;31(6):24650. doi: 10.1016/j.pt.2015.03.005.

14. Botelho MC, Vale N, Gouveia MJ, Rinaldi G, Santos J, Santos LL, et al Tumour-like phenotypes in urothelial cells after exposure to antigens from eggs of Schistosoma haematobium: an oestrogen-DNA adducts mediated pathway? Int J Parasitol. 2013;43(1):17-26. doi: 10.1016/j.ijpara.2012.10.023. [PubMed: 23260770].

15. Botelho MC, Soares R, Vale N, Ribeiro R, Camilo V, Almeida R, et al. Schistosoma haematobium: identification of new estrogenic molecules with estradiol antagonistic activity and ability to inactivate estrogen receptor in mammalian cells. Exp Parasitol. 2010;126(4):526-35. doi:10.1016/j.exppara.2010.06.012. [PubMed: 20547157].

16. Yabroff KR, Davis WW, Lamont EB, Fahey A, Topor M, Brown ML, et al. Patient time costs associated with cancer care. J Natl Cancer Inst. 2007;99(1):14-23. doi: 10.1093/jnci/djk001. [PubMed: 17202109].

17. Noyes K, Singer EA, Messing EM. Healthcare economics of bladder cancer: cost-enhancing and cost-reducing factors. Curr Opin Urol. 2008;18(5):533-9. doi: 10.1097/MOU.0b013e32830b8910. [PubMed 18670280].

18. Kulkarni GS, Alibhai SM, Finelli A, Fleshner NE, Jewett MA, Lopushinsky SR, et al. Cost-effectiveness analysis of immediate radical cystectomy versus intravesical Bacillus Calmette-Guerin therapy for highrisk, high-grade (T1G3) bladder cancer. Cancer. 2009;115(23):5450-9. doi: 10.1002/cncr.24634. [PubMed: 19685529].

19. Cooksley CD, Avritscher EB, Grossman HB, Sabichi AL, Dinney CP, Pettaway $C$, et al. Clinical model of cost of bladder cancer in the elderly. Urology. 2008;71(3):519-25. doi: 10.1016/j.urology.2007.10.056. [PubMed: 18342201].

20. Konety BR, Allareddy V. Influence of post-cystectomy complications on cost and subsequent outcome. J Urol. 2007;177(1):280-7. doi: 10.1016/j.juro.2006.08.074. [PubMed: 17162064] discussion 287.

21. Doehring E, Ehrich JH. [Effectiveness of praziquantel as an antihelmintic agent in the treatment of bilharziasis]. Monatsschr Kinderheilkd. 1986;134(5):282-4. [PubMed: 3088435].

22. Doehring E, Ehrich JH, Bremer HJ. Reversibility of urinary tract abnormalities due to Schistosoma haematobium infection. Kidney Int. 1986;30(4):582-5. [PubMed: 3097374].

23. Richter J. Evolution of schistosomiasis-induced pathology after therapy and interruption of exposure to schistosomes: a review of ultra- sonographic studies. Acta Trop. 2000;77(1):111-31. [PubMed: 10996127].

24. Richter J. The impact of chemotherapy on morbidity due to schistosomiasis. Acta Trop. 2003;86(2-3):161-83. [PubMed: 12745135].

25. Homeida MA, el Tom I, Nash T, Bennett JL. Association of the therapeutic activity of praziquantel with the reversal of Symmers' fibrosis induced by Schistosoma mansoni. Am J Trop Med Hyg. 1991;45(3):360-5. [PubMed: 1928571].

26. Nagi MA. Evaluation of a programme for control of schistosoma haematobium infection in Yemen. East Mediterr Health J. 2005;11(56):977-87. [PubMed:16761668].

27. Zhang Y, Koukounari A, Kabatereine N, Fleming F, Kazibwe F, Tukahebwa E, et al. Parasitological impact of 2-year preventive chemotherapy on schistosomiasis and soil-transmitted helminthiasis in Uganda. BMC Med. 2007;5:27. doi: 10.1186/1741-7015-5-27. [PubMed: 17767713].

28. Ndeffo Mbah ML, Gilbert JA, Galvani AP. Evaluating the potential impact of mass praziquantel administration for HIV prevention in Schistosoma haematobium high-risk communities. Epidemics. 2014;7:22-7. doi: 10.1016/j.epidem.2014.04.002. [PubMed: 24928666].

29. Ouedraogo H, Drabo F, Zongo D, Bagayan M, Bamba I, Pima T, et al. Schistosomiasis in school-age children in Burkina Faso after a decade of preventive chemotherapy. Bull World Health Organ. 2016;94(1):3745. doi: 10.2471/BLT.15.161885. [PubMed: 26769995].

30. van der Werf MJ, de Vlas SJ, Brooker S, Looman CW, Nagelkerke NJ, Habbema JD, et al. Quantification of clinical morbidity associated with schistosome infection in sub-Saharan Africa. Acta Trop. 2003;86(2-3):125-39. [PubMed: 12745133].

31. Hotez PJ, Fenwick A, Kjetland EF. Africa's 32 cents solution for HIV|AIDS. PLoS Negl Trop Dis. 2009;3(5):e430. doi: 10.1371/journal.pntd.0000430. [PubMed: 19479041].

32. King CH. Parasites and poverty: the case of schistosomiasis. Acta Trop. 2010;113(2):95-104. doi: 10.1016/j.actatropica.2009.11.012. [PubMed: 19962954].

33. Gabrielli AF, Toure S, Sellin B, Sellin E, Ky C, Ouedraogo H, et al. A combined school- and community-based campaign targeting all school-age children of Burkina Faso against schistosomiasis and soil-transmitted helminthiasis: performance, financial costs and implications for sustainability. Acta Trop. 2006;99(2-3):234-42. doi: 10.1016/j.actatropica.2006.08.008. [PubMed: 16997268].

34. Fenwick A. Waterborne infectious diseases-could they be consigned to history? Science. 2006;313(5790):1077-81. doi: 10.1126/science.1127184. [PubMed:16931751].

35. Poggensee G, Kiwelu I, Saria M, Richter J, Krantz I, Feldmeier H. Schistosomiasis of the lower reproductive tract without egg excretion in urine. Am J Trop Med Hyg. 1998;59(5):782-3. [PubMed: 9840597].

36. Richter J, Bode JG, Blondin D, Kircheis G, Kubitz R, Holtfreter MC, et al. Severe liver fibrosis caused by Schistosoma mansoni: management and treatment with a transjugular intrahepatic portosystemic shunt. Lancet Infect Dis. 2015;15(6):731-7. doi: 10.1016/S1473-3099(15)70009-5. [PubMed: 25769268].

37. Koukounari A, Donnelly CA, Sacko M, Keita AD, Landoure A, Dembele $\mathrm{R}$, et al. The impact of single versus mixed schistosome species infections on liver, spleen and bladder morbidity within Malian children pre-and post-praziquantel treatment. BMC Infect Dis. 2010;10:227. doi: 10.1186/1471-2334-10-227. [PubMed: 20670408]. 